\title{
Some results on zeros of palindromic and perturbed polynomials of even degree
}

\author{
Vanessa Botta \\ Depto de Matemática e Computação, FCT, UNESP, \\ 15054-000, Presidente Prudente, SP \\ E-mail: botta@fct.unesp.br.
}

\begin{abstract}
In this paper we give necessary and sufficient conditions for all zeros of palindromic polynomial of even degree $R(z)=1+\lambda\left(z+z^{2}+\ldots+z^{n-1}\right)+z^{n}$, with $\lambda \in \mathbb{R}$, to be on the unit circle and we find $\gamma \in \mathbb{R}$ for which $S(z)=R(z)+\gamma z^{n}$ has all its zeros inside or on the unit circle .
\end{abstract}

Keywords: Palindromic Polynomials, Zeros of Polynomials, Perturbed Polynomials

Let $P(z)=a_{0}+a_{1} z+\ldots+a_{n} z^{n}$ be a polynomial of degree $n, n \geq 1, a_{i} \in \mathbb{R}, i=0, \ldots, n$. Then $P$ is palindromic if $a_{i}=a_{n-i}$, for every $i=0,1, \ldots, n$. In this paper we give necessary and sufficient conditions for all zeros of palindromic polynomial of even degree $n, n \geq 1$, $R(z)=1+\lambda\left(z+z^{2}+\ldots+z^{n-1}\right)+z^{n}$, with $\lambda \in \mathbb{R}$, to lie on the unit circle. Furthermore, we prove that the polynomial $S(z)=R(z)+\gamma z^{n}$, with $\gamma \geq \lambda-2(\gamma>0, \lambda \geq 0)$, has all its zeros in the closed unit disc. More details can be found in [1, 4].

\section{Classical results}

Theorem 1.1 (Eneström-Kakeya, real coefficients case). Let $P(z)=\sum_{i=0}^{n} a_{i} z^{i}$ be a polynomial such that $0<a_{0} \leq a_{1} \leq \ldots \leq a_{n}$. Then, $P(z)$ has all its zeros in the closed unit disc.

Definition 1.2. Let the polynomial $P(z)=\sum_{i=0}^{n} a_{i} z^{i}, a_{i} \in \mathbb{R}$. Define the associated polynomial

$$
P^{*}(z)=z^{n} P\left(\frac{1}{z}\right)=a_{0} z^{n}+a_{1} z^{n-1}+\ldots+a_{n}=a_{0} \prod_{j=1}^{n}\left(z-z_{j}^{*}\right),
$$

whose zeros $z_{k}^{*}$ are the inverses of the zeros $z_{k}$ of $P(z)$, that is, $z_{k}^{*}=\frac{1}{\overline{z_{k}}}$.

Definition 1.3. If $P(z)=P^{*}(z)$, that is, $P(z)=z^{n} P\left(\frac{1}{z}\right)$, the polynomial $P(z)$ is said to be palindromic.

It is clear that if $P(z)=\sum_{i=0}^{n} a_{i} z^{i}, a_{i} \in \mathbb{R}, i=0, \ldots, n$, is palindromic, then $a_{i}=a_{n-i}$, $i=0,1, \ldots, n$, as we mentioned above.

Definition 1.4. Given $P(z)$ with real coefficients, the sequence of polynomials $P_{j}(z)$ is defined by:

$$
P_{j}(z)=\sum_{k=0}^{n-j} a_{k}^{(j)} z^{k}, \text { where } P_{0}(z)=P(z) \text { and }
$$




$$
P_{j+1}(z):=a_{0}^{(j)} P_{j}(z)-a_{n-j}^{(j)} P_{j}^{*}(z), j=0,1, \ldots, n-1,
$$

with $P_{0}^{*}(z)=P^{*}(z)$.

From (1.1), the coefficients of $P_{j+1}(z)$ satisfy the recurrence relation

$$
a_{k}^{(j+1)}=a_{0}^{(j)} a_{k}^{(j)}-a_{n-j}^{(j)} a_{n-j-k}^{(j)}, k=0,1, \ldots n-j \text { and } j=0,1, \ldots n .
$$

Definition 1.5. For each polynomial $P_{j}(z)$ we shall denote the constant term $a_{0}^{(j)}$ by $\delta_{j}$ and

$$
\delta_{j+1}=a_{0}^{(j+1)}=\left|a_{0}^{(j)}\right|^{2}-\left|a_{n-j}^{(j)}\right|^{2}, j=0,1, \ldots, n-1 .
$$

Lemma 1.6. If $P_{j}$ has $p_{j}$ zeros in $|z|<1$ and if $\delta_{j+1} \neq 0$, then $P_{j+1}$ has

$$
p_{j+1}=\left\{\begin{array}{lll}
p_{j}, & \text { if } \quad \delta_{j+1}>0 \\
n-j-p_{j}, & \text { if } \quad \delta_{j+1}<0
\end{array}\right.
$$

zeros in $|z|<1$. Furthermore, $P_{j+1}$ has the same zeros on $|z|=1$ as $P_{j}$.

The proof of this lemma may be found in Marden [3], p. 195.

The next result is due to Schur $[5,6]$ and the proof follows from Lemma 1.6.

Lemma 1.7. If $0<\left|a_{0}\right|<\left|a_{n}\right|$, then $P(z)$ has all its zeros in the closed unit disc if, and only if, $P_{1}^{*}(z)$ has all its zeros in the closed unit disc.

Using the same notation presented in [2], let $\mathbf{a}=\left(a_{1}, a_{2}, \ldots, a_{n-1}\right) \in \mathbb{R}^{n-1}$ and $L: \mathbb{R}^{n-1} \rightarrow \mathbb{R}$ be a function defined by

$$
L(\mathbf{a}):=\min _{y \in \mathbb{R}} \sum_{j=1}^{n-1}\left|a_{j}-y\right| .
$$

With a permutation $\sigma$ on $\{1,2, \ldots, n-1\}$ for which $a_{\sigma(1)} \leq a_{\sigma(2)} \leq \ldots \leq a_{\sigma(n-1)}$ one has: if $n$ is even, then $L(\mathbf{a})=\sum_{j=1}^{n-1}\left|a_{j}-a_{\sigma(n / 2)}\right|$; if $n$ is odd, then $L(\mathbf{a})=\sum_{j=1}^{n-1}\left|a_{j}-y\right|$ for every $y$ in a closed interval $\left[a_{\sigma(\lfloor n / 2\rfloor)}, a_{\sigma(\lceil n / 2\rceil)}\right]$, where $\lfloor t\rfloor:=\max (-\infty, t] \cap \mathbb{Z}$ and $\lceil t\rceil:=\min [t, \infty) \cap \mathbb{Z}$. In addition, considering $\bar{m}(\mathbf{a})$ (resp. $\underline{m}(\mathbf{a}))$ defined by $\bar{m}(\mathbf{a}):=a_{\sigma(\lceil n / 2\rceil)}\left(\operatorname{resp} . \underline{m}(\mathbf{a}):=a_{\sigma(\lfloor n / 2\rfloor)}\right)$ then $\bar{m}(\mathbf{a})=\underline{m}(\mathbf{a})$ when $n$ is even.

Theorem 1.8. Let $P(z)=\sum_{i=0}^{n} a_{i} z^{i}$ be a palindromic polynomial of degree $n$ with $a_{n}>0$, and let $\mathbf{a}=\left(a_{1}, a_{2}, \ldots, a_{n-1}\right)$.

1. Suppose $\underline{m}(\mathbf{a})+L(\mathbf{a}) \leq 2 a_{n}$.

(a) If $P(1) \geq 0$, then all zeros of $P$ lie on the unit circle. In this case, there are at least two zeros of the form $e^{i \theta}$ with $-\frac{2 \pi}{n} \leq \theta \leq \frac{2 \pi}{n}$.

(b) If $P(1)<0$, then $P$ has real zeros $\beta>1$ and $\beta^{-1}$ and the other zeros lie on the unit circle.

2. Suppose $\bar{m}(\mathbf{a}) \geq L(\mathbf{a})+2 a_{n}$. Then one of the following holds:

(a) All the zeros of $P$ lie on the unit circle. When $n$ is odd, there are three or five zeros of the form $e^{i \theta}$ with $\frac{(n-1) \pi}{n} \leq \theta \leq \frac{(n+1) \pi}{n}$. When $n$ is even, -1 is a zero with multiplicity 2 or 4.

(b) $P$ has real zeros $\beta<-1$ and $\beta^{-1}$ and the other zeros lie on the unit circle.

The proof of this result may be found in [2]. 


\section{Main Results}

Theorem 2.1. The zeros of the polynomial $R(z)=1+\lambda\left(z+z^{2}+\ldots+z^{n-1}\right)+z^{n}, \lambda \in \mathbb{R}$, of even degree $n>1$, lie on the unit circle if and only if $-\frac{2}{n-1} \leq \lambda \leq 2$.

Proof. From Theorem 1.8, a $=(\lambda, \lambda, \ldots, \lambda), \bar{m}(\mathbf{a})=\underline{m}(\mathbf{a})=\lambda$ and $L(\mathbf{a})=0$.

If $\underline{m}(\mathbf{a})+L(\mathbf{a}) \leq 2$, i.e., $\lambda \leq 2$, as $R(1)=2+(n-1) \lambda \geq 0$ when $\lambda \geq-\frac{2}{n-1}$, from item (1) (a) of Theorem 1.8 follows that all zeros of $R(z)$ lie on the unit circle when $-\frac{2}{n-1} \leq \lambda \leq 2$.

Furthermore, if $\underline{m}(\mathbf{a})+L(\mathbf{a})=\lambda \leq 2$ and $R(1)=2+(n-1) \lambda<0$, i.e., $\lambda<-\frac{2}{n-1}, R(z)$ has one real root in $(1, \infty)$. In fact,

$$
\lim _{z \rightarrow 1} R(z)=2+(n-1) \lambda<0 \text { and } \lim _{z \rightarrow+\infty} R(z)>0,
$$

that is, there is a signal change of $R(z)$ in $(1, \infty)$. This case is described in item (1) (b) of Theorem 1.8.

If $\lambda>2(\bar{m}(\mathbf{a})>L(\mathbf{a})+2), R(z)$ has one real root in $(-\infty,-1)$. In fact,

$$
\lim _{z \rightarrow-\infty} R(z)>0 \text { and } \lim _{z \rightarrow-1} R(z)=2-\lambda<0,
$$

that is, there is a signal change of $R(z)$ in $(-\infty,-1)$. Observe that this case is described in item (2) (b) of Theorem 1.8 .

So, for $n$ even, we prove that the zeros of $R(z)$ lie on the unit circle if, and only if, $-\frac{2}{n-1} \leq \lambda \leq 2$.

Remark 2.2. If $n$ is even and $\lambda=2$, we have $R(-1)=0$ and $z=-1$ is a zero of multiplicity 2 of $R(z)$, as described in item (2) (a) of Theorem 1.8.

Theorem 2.3. The perturbed polynomial

$$
S(z)=R(z)+\gamma z^{n}=1+\lambda\left(z+z^{2}+\ldots+z^{n-1}\right)+(1+\gamma) z^{n},(\lambda \geq 0, \gamma>0, n \text { even })
$$

has all zeros in the closed unit disc if $\gamma \geq \lambda-2$ and has at least one zero outside the closed unit disc if $\gamma<\lambda-2$.

Proof. For $\lambda=0$, we have $S(z)=1+(1+\gamma) z^{n}$ and the proof is immediate.

From here, we consider $\lambda>0$.

We write the polynomials $S(z)$ and $S_{1}(z)$ in the form

$$
S(z)=s_{n} z^{n}+s_{n-1} z^{n-1}+\ldots+s_{0},
$$

where $s_{n}=1+\gamma, s_{i}=\lambda, i=1, \ldots, n-1$, and $s_{0}=1$, and

$$
S_{1}(z)=s_{n-1}^{(1)} z^{n-1}+s_{n-2}^{(1)} z^{n-2}+\ldots+s_{0}^{(1)},
$$

where the coefficients $s_{k}^{(1)}, k=0,1, \ldots, n-1$, are defined by equation 1.2 using $j=0$. So,

$$
s_{k}^{(1)}=s_{0} s_{k}-s_{n} s_{n-k} .
$$

Substituting the values of $s_{k}, k=0, \ldots, n$, we have

$$
s_{n-1}^{(1)}=s_{n-2}^{(1)}=\ldots=s_{1}^{(1)}=-\gamma \lambda<0 \text { and } s_{0}^{(1)}=-\gamma(\gamma+2)<0 .
$$

Note that, as $\gamma>0,0<1<1+\gamma$, i.e., $0<s_{0}<s_{n}$, Lemma 1.7 can be applied to conclude that the zeros of $S(z)$ lie in the closed unit disc if and only if the zeros of $S_{1}^{*}(z)$ do.

Observe that

$$
-S_{1}^{*}(z)=\left|s_{n-1}^{(1)}\right|+\left|s_{n-2}^{(1)}\right| z+\ldots+\left|s_{1}^{(1)}\right| z^{n-2}+\left|s_{0}^{(1)}\right| z^{n-1} .
$$


If $\left|s_{0}^{(1)}\right| \geq\left|s_{1}^{(1)}\right|>0$, the coefficients of $-S_{1}^{*}(z)$ are ordered and by the Eneström-Kakeya Theorem, the zeros of $-S_{1}^{*}(z)$ lie in $|z| \leq 1$. As the zeros of $S_{1}^{*}(z)$ and $-S_{1}^{*}(z)$ are the same, the zeros of $S_{1}^{*}(z)$ lie in $|z| \leq 1$ too.

But

$$
\left|s_{0}^{(1)}\right|-\left|s_{1}^{(1)}\right|=\gamma(\gamma+2-\lambda) \geq 0 .
$$

Then, $\left|s_{0}^{(1)}\right| \geq\left|s_{1}^{(1)}\right|$ is equivalent to $\gamma \geq \lambda-2$.

So, for $\gamma \geq \lambda-2, S(z)$ has all its zeros in $|z| \leq 1$.

Now we prove that, if $\gamma<\lambda-2, S(z)$ has at least one zero outside the unit disc.

As

$$
\left|s_{0}^{(1)}\right|-\left|s_{n-1}^{(1)}\right|=\gamma(\gamma+2-\lambda),
$$

$\left|s_{0}^{(1)}\right|<\left|s_{n-1}^{(1)}\right|$ is equivalent to $\gamma<\lambda-2$.

By the Vieta's formula, we have

$$
\zeta_{1} \zeta_{2} \ldots \zeta_{n-1}=(-1)^{n-1} \frac{s_{n-1}^{(1)}}{s_{0}^{(1)}},
$$

where $\zeta_{i}, i=1, \ldots, n-1$, are the zeros of $S_{1}^{*}(z)$.

So, if $\gamma<\lambda-2$, follows that

$$
\left|\zeta_{1} \zeta_{2} \ldots \zeta_{n-1}\right|=\left|\frac{s_{n-1}^{(1)}}{s_{0}^{(1)}}\right|>1 .
$$

Then, at least one zero of $S_{1}^{*}(z)$ lie outside the unit disc and, consequently, $S(z)$ has at least one zero outside the unit disc.

Remark 2.4. For $\gamma=0$ we have $S(z)=R(z)$ and the zeros of $S(z)$ lie on the unit circle under the conditions of Theorem 2.1.

\section{Numerical Examples}

Example 3.1. Let us consider the polynomial $R(z)=1+\frac{5}{3}\left(z+z^{2}+z^{3}\right)+z^{4}$. Figure 1 displays the zeros of $R(z)$ (represented by $\bullet$ ) and $S(z)$ for $\gamma=0.5$ (represented by $*$ ). Note that the conditions of Theorem 2.1 are satisfied and the zeros of $R(z)$ lie on the unit circle. From Theorem 2.3 the zeros of the perturbed polynomial $S(z)$, for all $\gamma \geq 0$, lie inside or on the unit circle.

Example 3.2. Let us consider the polynomial $R(z)=1+2 z+2 z^{2}+2 z^{3}+2 z^{4}+2 z^{5}+z^{6}$. Figure 2 displays the zeros of $R(z)$ (represented by $\bullet$ ) and $S(z)$ for $\gamma=0.8$ (represented by ${ }^{*}$ ). The conditions of Theorem 2.1 are satisfied and the zeros of $R(z)$ lie on the unit circle (from Remark $2.2, z=-1$ is a zero of multiplicity 2 of $R(z)$ ). From Theorem 2.3 the zeros of the perturbed polynomial $S(z)$, for all $\gamma \geq 0$, lie inside or on the unit circle. 


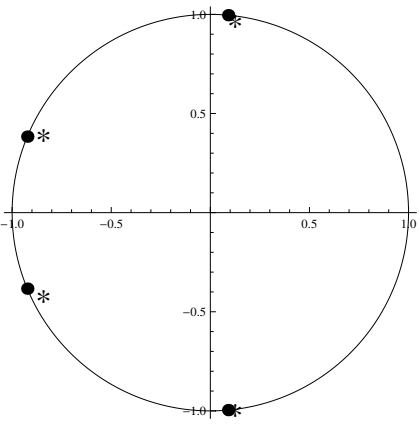

Figure 1: Zeros of $S(z)=1+$ $\frac{5}{3}\left(z+z^{2}+z^{3}\right)(1+\gamma) z^{4}$ for $\gamma=0$ (dots) and $\gamma=0.5$ (stars).

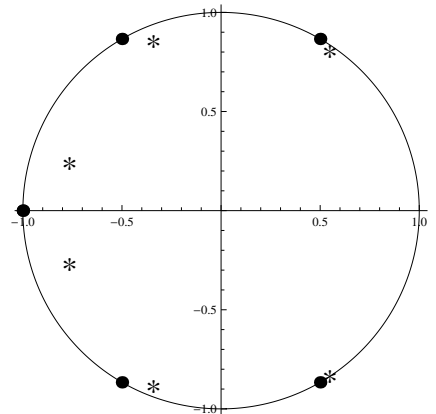

Figure 2: Zeros of $S(z)=1+2 z+2 z^{2}+$ $2 z^{3}+2 z^{4}+2 z^{5}+(1+\gamma) z^{6}$ for $\gamma=0$ (dots) and $\gamma=0.8$ (stars).

Example 3.3. Let us consider the polynomial $R(z)=1+4\left(z+z^{2}+z^{3}\right)+z^{4}$. Figure 3 displays the zeros of $R(z)$ (represented by $\bullet$ ) and $S(z)$ for $\gamma=2$ (represented by ${ }^{*}$ ) and $\gamma=4$ (represented by + ). As $\lambda=4>2$, from Theorem $2.1 R(z)$ has one real zero in $(-\infty,-1)$. From Theorem 2.3 , the zeros of $S(z)$ lie inside or on the unit circle when $\gamma \geq 2$ and for $0<\gamma<2, S(z)$ has at least one zero outside the unit circle.

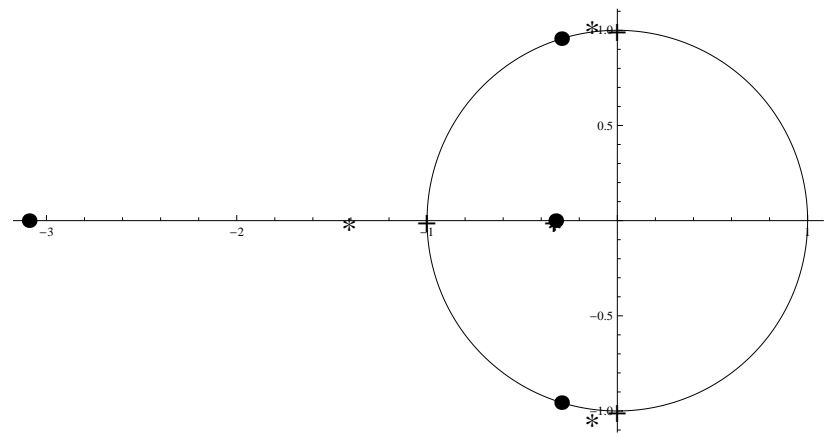

Figure 3: Zeros of $S(z)=1+4\left(z+z^{2}+z^{3}\right)+(1+\gamma) z^{4}$ for $\gamma=0($ dots $), \gamma=1$ (stars) and $\gamma=2$ (plus).

\section{Acknowledgment}

Grant \#2013/08012-8, São Paulo Research Foundation (FAPESP).

\section{References}

[1] V. Botta, L. F. Marques, M. Meneguette, Palindromic and perturbed polynomials: zeros location, Acta Math. Hungar., Published online: 11 Dec 2013.

[2] D. Kwon, Reciprocal polynomials with all but two zeros on the unit circle, Acta Math. Hungar., 134 (4) (2012), 472-480.

[3] M. Marden, "Geometry of Polynomials", American Mathematical Society (Providence, 1966).

[4] L. F. Marques, Zeros de polinômios perturbados, Master Thesis (Presidente Prudente, 2013).

[5] J. Schur, Über Potenzreihen, die in Innern des Einheitskreises beschränkt sind, J. Reine Angew. Math., 147 (1917), 205-232. 
Proceeding Series of the Brazilian Society of Applied and Computational Mathematics, Vol. 3, N. 1, 2015.

[6] J. Schur, Über Polynome, die nur in Innern des Einheitkreis verschwinden, J. Reine Angew. Math., 148 (1918), 122-145. 